\title{
Distance to the solar wind termination shock and the source flux of anomalous cosmic rays during 1986-1988
}

\author{
A. C. Cummings and E. C. Stone \\ Space Radiation Laboratory, California Institute of Technology, Pasadena \\ W. R. Webber \\ Department of Physics, New Mexico State University, Las Cruces
}

\begin{abstract}
We use observations of the intensity of anomalous cosmic ray helium and oxygen in the outer heliosphere near the last solar minimum period, in conjunction with solar observations of the tilt of the neutral current sheet, to estimate that the solar wind termination shock was at $67 \pm 5 \mathrm{AU}$ during the time period 1986.7-1988.5. We find the source flux of anomalous cosmic rays at the shock to be $1.7 \pm 0.3\left(\mathrm{~m}^{2} \mathrm{~s} \mathrm{sr}\right.$ $\mathrm{MeV} /$ nucleon $)^{-1}$ for 7.1-17.1 MeV/nucleon anomalous cosmic ray oxygen and $4.3 \pm$

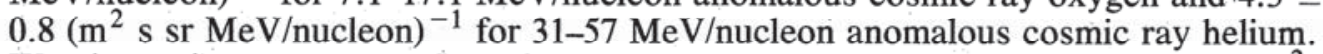
We also estimate a source flux of anomalous cosmic ray hydrogen of $2.3 \pm 0.6\left(\mathrm{~m}^{2} \mathrm{~s}\right.$ sr $\mathrm{MeV})^{-1}$ at $134 \mathrm{MeV}$. The resulting energy density of anomalous cosmic rays at the solar wind termination shock is $\sim 0.03 \mathrm{eV} / \mathrm{cm}^{3}$, approximately $2 \%$ of the energy density of the solar wind at $67 \mathrm{AU}$.
\end{abstract}

\section{Introduction}

Anomalous cosmic rays (ACRs) are thought to originate as interstellar neutral atoms that drift into the heliosphere and become ionized [Fisk et al., 1974] and accelerated to energies of several hundred $\mathrm{MeV}$ at the termination shock of the solar wind [Pesses et al., 1981]. Because the ionized atoms in the local interstellar medium are deflected around the heliosphere by the interplanetary magnetic field, only those atoms with high first ionization potentials are expected to flow into the heliosphere to become the ACR component. Indeed, only $\mathrm{He}, \mathrm{N}, \mathrm{O}, \mathrm{Ne}$, and $\mathrm{Ar}$ are found in significant abundance in the ACR component [Cummings and Stone, 1990]. All of these atoms have first ionization potentials above that of $\mathrm{H}$. Evidence for anomalous cosmic ray hydrogen has been presented [Christian et al., 1988, 1990; Christian, 1989; Mewaldt, 1990] but its existence remains controversial [Lopate and McKibben, 1991; Reinecke and Moraal, 1992; Seo et al., 1993]. Carbon appears to be an ACR species that is present in a very small quantity, consistent with its relatively low first ionization potential and correspondingly low abundance of neutral interstellar C. Recently, the prediction that the ACRs should be singly ionized has been confirmed by observations [Adams et al., 1991]. In addition, $\mathrm{H}, \mathrm{He}, \mathrm{N}, \mathrm{O}$, and $\mathrm{Ne}$ pickup ions, which are believed to become the seed particles of the anomalous component, have been observed in the solar wind [Möbius et al., 1985; Gloeckler et al., 1993; Geiss et al., 1994].

Although none of the spacecraft in the outer heliosphere, Pioneer 10 (P10), Pioneer 11 (P11), Voyager 1 (V1), and Voyager 2 (V2), have crossed the termination shock, they observe a positive radial gradient of the ACRs [Cummings et al., 1990a] consistent with having the effective "source" of

Copyright 1994 by the American Geophysical Union.

Paper number 94JA00551.

0148-0227/94/94JA-00551\$05.00 these particles at the termination shock, beyond the location of the most distant spacecraft.

Recently, we have employed two different methods to make estimates of the distance to the termination shock using observations of the radial gradient of ACR oxygen in the outer heliosphere and of the tilt of the neutral sheet. The first method [Cummings et al., 1993a] compared so-called "shifted" and "instantaneous" gradients and resulted in an estimate of $62_{-7}^{+5} \mathrm{AU}$ for the period 1986.5-1988.5. In the second method [Cummings et al., 1993b] the same data set was used, but the distance to the shock and the flux at the shock were deduced from only the instantaneous gradients and the neutral sheet tilt observations. The distance estimate from this second analysis, $62 \pm 2 \mathrm{AU}$, was similar to the first. The estimated flux of ACR oxygen at the shock was $1.27 \pm 0.04\left(\mathrm{~m}^{2} \mathrm{~s} \text { sr MeV/nucleon }\right)^{-1}$ in the energy interval 7.1-17.1 MeV/nucleon. The analysis method used in the present paper is nearly identical to that in the latter work. The flux versus time data set has been reduced by two 52-day periods to remove the effects of a transient decrease in the cosmic ray fluxes. More importantly, we have added ACR helium to the analysis as a test of the applicability of the method.

\section{Analysis Approach}

The analysis method is based upon a simple model with three assumptions: (1) the source flux of ACRs is constant at the shock during the $\sim 2$-year period of the observations, (2) the shock location does not vary, and (3) we can use the observed correlation of the radial gradient of ACRs between V2 at $\sim 24$ and P10 at $\sim 42$ AU with the estimated tilt of the neutral current sheet between the two spacecraft [Cummings et al., 1990b] to extrapolate fluxes beyond the radial position of P10. Because the time history of the tilt angle is known, this latter assumption allows a straightforward extrapolation of the flux of ACRs beyond the point of observation. 

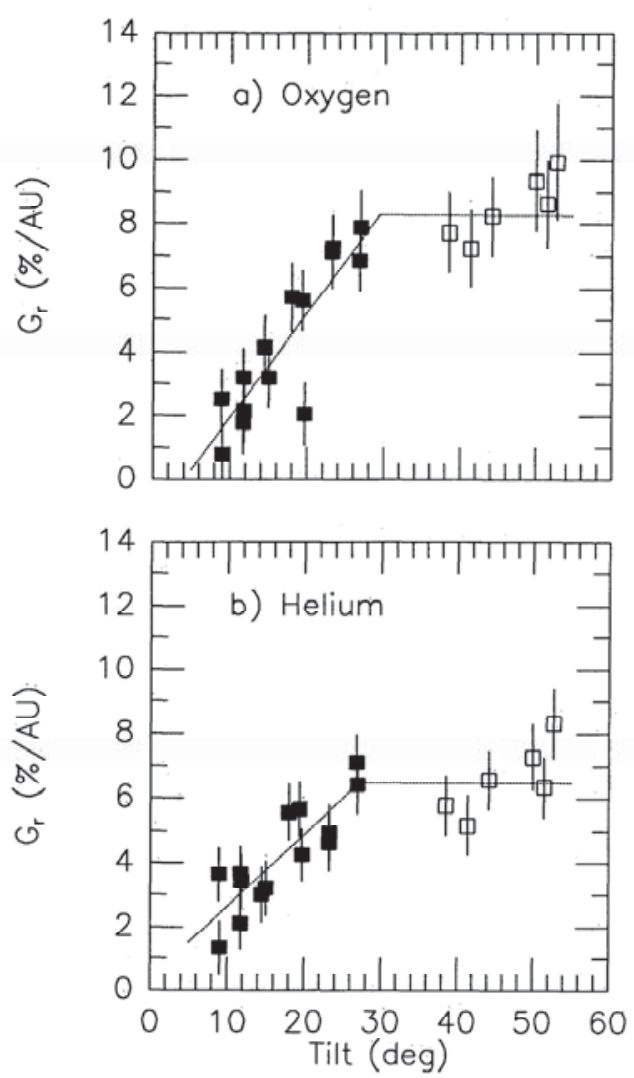

Figure 1. (a) Radial gradient of 7.1-17.1 MeV/nucleon ACR oxygen between the radial positions of V2 and P10 versus neutral current sheet tilt angle for 20 52-day time periods between 1986.1 and 1989.4. The two lines are least squares fits to the data (sloping line uses data represented as closed squares; horizontal line uses open squares). (b) Same as Figure 1a except for 30.5-56.5 MeV/nucleon ACR helium.

The observed correlations of the neutral sheet tilt angle $\alpha$, with the local radial gradient of ACR helium and oxygen, $G_{R}$, is shown in the two panels of Figure 1. The local radial gradient was measured between V2 and P10 from

$$
G_{R}=\frac{\ln \left(I_{\mathrm{P} 10} / I_{\mathrm{V} 2}\right)}{\Delta R}
$$

where $I_{\mathrm{P} 10}$ and $I_{\mathrm{V} 2}$ are the ACR fluxes at P10 and V2, respectively, and $\Delta R$ is the distance between the two spacecraft. The correlations in Figure 1 are excellent when the tilt angle is $\$ 30^{\circ}$. The average tilt angle of the neutral current sheet, $\alpha$, between P10 and V2 has been estimated by "shifting" the near-Sun tilt data [Hoeksema, 1992] to the midpoint between the spacecraft at $400 \mathrm{~km} / \mathrm{s}$, the typical speed of the solar wind.

During the solar minimum period near 1987 the drift of positively charged particles is inward into the heliosphere along the neutral current sheet (see Jokipii [1990]). Cummings et al. [1990b] demonstrated that the observed time variation of the local gradient between V2 and P10 was consistent with particles drifting and diffusing along a neutral sheet whose length was proportional to the tilt angle. The local radial gradient observations were fit quite well by a simple model in which the perpendicular diffusion coefficient $\kappa_{\perp}$ is proportional to the radius $R$ and the gradient is specified along the neutral sheet.

With the assumptions that both the shape of the neutral sheet and $\kappa_{\perp}$ scale as $R$ the dependence of $G_{R}$ on $\alpha$ should be independent of radius. Note that we do not require that the neutral sheet have a sinusoidal shape with tilt angle $\alpha$, only that the distance along the sheet for a given radial separation be proportional to the tilt angle and the radius $R$. Thus the best fit lines shown in the two panels of Figure 1:

oxygen

$$
G_{R}(\% / \mathrm{AU})=0.3265 \alpha-1.328 \quad \alpha<29.4^{\circ}
$$

and helium

$$
G_{R}(\% / \mathrm{AU})=0.2247 \alpha+0.3746 \quad \alpha<27.1^{\circ}
$$

allow us to use the temporal dependence of the tilt angle $\alpha$ of the outwardly propagating neutral sheet to estimate $G_{R}$ at distances beyond P10. Because the dependences in (2) and (3) were derived for a radial separation of $\sim 18 \mathrm{AU}$ between P10 and V2 when P10 was between $\sim 39$ and $\sim 44 \mathrm{AU}$, it seems reasonable to expect the relationship to apply to distances $\gtrsim 18 \mathrm{AU}$ beyond P10, that is, at least out to 60 or $70 \mathrm{AU}$.

If at a given time $t$ the local gradients $G_{N}$ are known out to the termination shock, then the observed intensity $I_{\mathrm{V} 2}(t)$ can be related to the source intensity $I_{\text {shock }}(t)$ by

$$
I_{\text {shock }}(t)=I_{\mathrm{V} 2}(t) \prod_{N=1}^{N=N_{\max }} \exp \left[G_{N}(t) \Delta R\right]
$$

where $\Delta R=V_{\mathrm{Sw}} \Delta t=6.3 \mathrm{AU}$ is the radial range interval corresponding to the minimum time interval $(\Delta t=27.3 d)$ for which $\alpha$ is known, $N_{\max }=\left[R_{\text {shock }}-R_{\mathrm{V} 2}(t)\right] / \Delta R$, and $G_{N}(t)$ is the estimated local gradient in the $N$ th radial range interval beyond V2. Using (2) and (3), (4) can be rewritten as

$$
I_{\text {shock }}(t)=I_{\mathrm{V} 2}(t) \prod_{N=1}^{N=N_{\max }} \exp \left\{\left[a \alpha_{\mathrm{V} 2}(t-N \Delta t)+b\right] \Delta R\right\}
$$

where $a$ and $b$ are 0.003265 and -0.01328 for oxygen and 0.002247 and 0.003746 for helium, respectively, and $\alpha_{\mathrm{V} 2}(t-$ $N \Delta t)$ is the time dependent tilt angle of the neutral current sheet at V2 (see Figure 2). As described below, we use (5) to calculate $I_{\text {shock }}$ for different assumed shock locations for 13 52-day time intervals between 1986.7 and 1988.5 using both ACR oxygen and ACR helium observations.

\section{Observations}

In Figure 3 we show the time histories of the ACR helium and oxygen fluxes at both $\mathrm{V} 2$ and P10 from the cosmic ray experiments on each spacecraft [Stone et al., 1977; McDonald et al., 1977] for the period 1986.7-1988.5. The four curves in each panel of Figure 3 are calculated from (5) above for oxygen and helium and correspond to the estimated flux at P10, $62 \mathrm{AU}, 75 \mathrm{AU}$, and $100 \mathrm{AU}$. For both helium and oxygen the calculated P10 curve is in good agreement with the observed flux profile; the small differences between the model and the data are due to using (2) and (3) to approximate the observed gradients. The other 


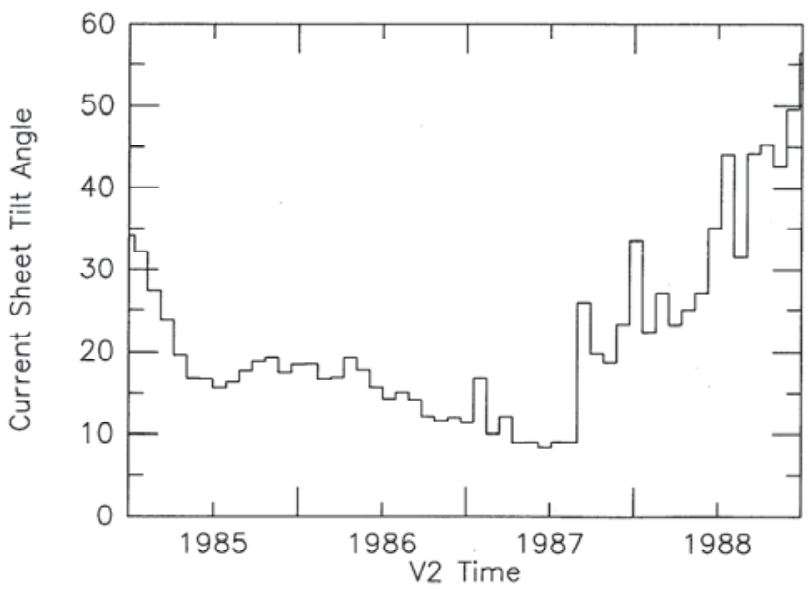

Figure 2. Estimated tilt of neutral current sheet at V2. The tilts observed at the Sun [Hoeksema, 1992] have been shifted to the position of V2 using a convection velocity of $400 \mathrm{~km} / \mathrm{s}$.

curves represent flux estimates for several candidate shock positions. If we assume that the flux at the shock is constant during this period, then the curve with the least time dependence in Figure 3 is the best candidate for the location of the shock. Although none of the curves are precisely
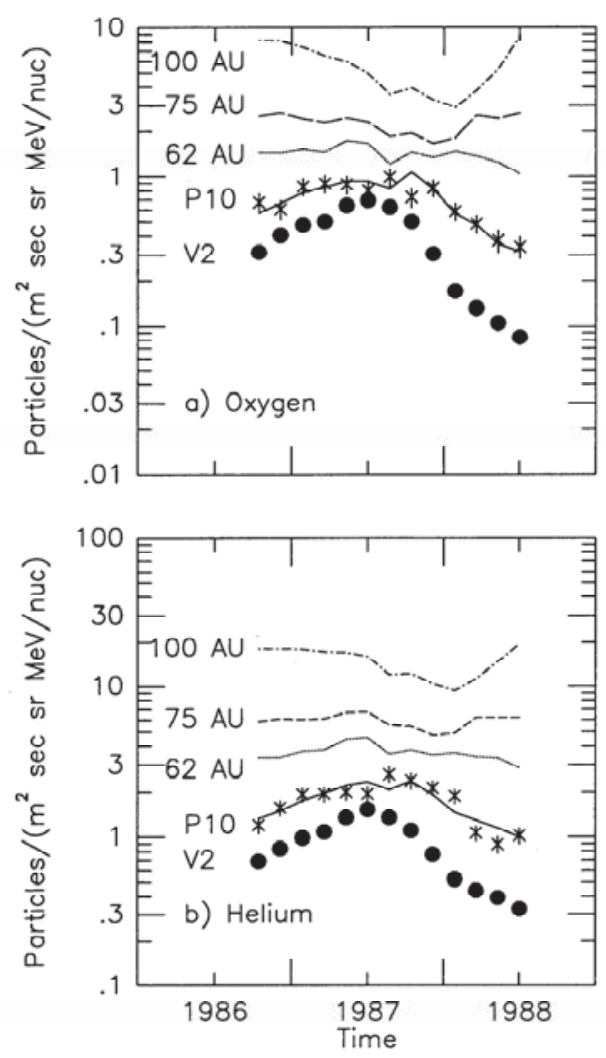

Figure 3. (a) The 52-day average fluxes of 7.1-17.1 MeV/ nucleon ACR oxygen at $\mathrm{P} 10$ and $\mathrm{V} 2$ versus time. The curves are extrapolated fluxes from V2 as described in the text. The solid line is the estimated flux at P10 versus time. The other curves represent the flux at several candidate shock locations. (b) Same as Figure $3 \mathrm{a}$ except for 30.5-56.5 MeV/ nucleon ACR helium.
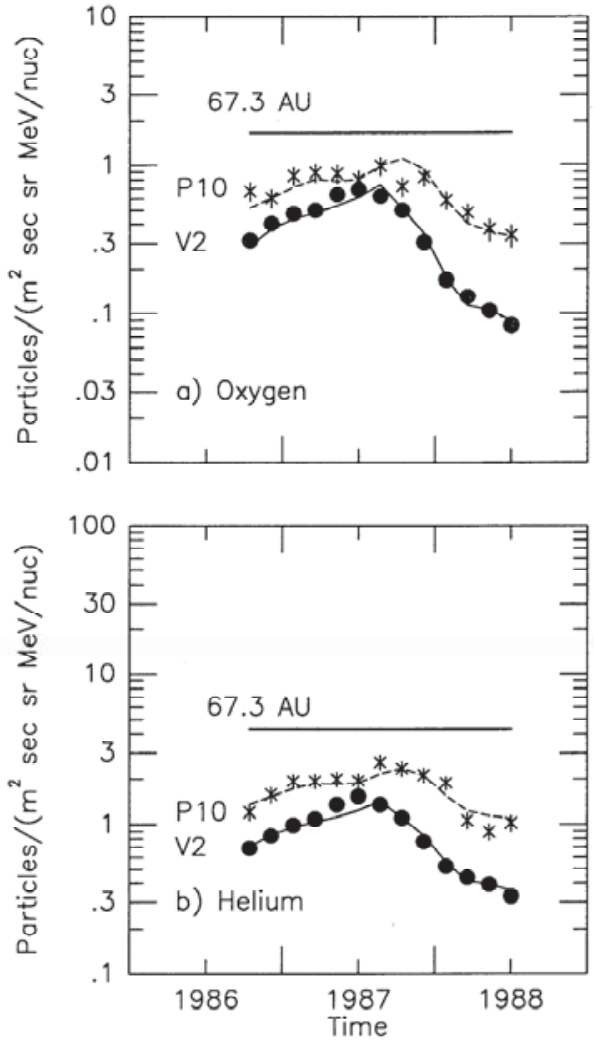

Figure 4. (a) The 52-day average fluxes of 7.1-17.1 MeV/ nucleon ACR oxygen at $\mathrm{P} 10$ and $\mathrm{V} 2$ versus time. The indicated shock flux and location have been obtained by simultaneously fitting the oxygen and helium data at P10 and V2 as shown by the dashed and solid curves in both panels. (b) Same as Figure 4a except for 30.5-56.5 MeV/nucleon ACR helium.

independent of time, it appears that the shock distance is between 62 and $75 \mathrm{AU}$, based on this criterion.

This bootstrap approach can also be applied in reverse by assuming a constant flux value at a fixed shock location and calculating the flux at P10 and V2 by using (5) along the tilt steps between the shock and the spacecraft. These calculated curves can be compared to the observations in a least squares sense to determine both the best choice of shock location and flux at the shock. In Figure 4 we show the result of requiring the best simultaneous fit to the helium and oxygen data sets; that is, we required the shock distance to be the same in both cases, but we allowed the flux of oxygen and helium to be different at the shock. The statistical errors for the observed fluxes were increased by adding in quadrature an assumed systematic error of $10 \%$ in order to account for the estimated systematic error in the dependence of $G_{R}$ on $\alpha$. The result of this fit is that the shock distance is 67.3 AU. Note that there are only three free parameters in this fit, the shock distance and the flux of ACR oxygen and helium at the shock. Independent fits of the oxygen and helium fluxes yield best fit shock locations of 64.5 and 70.9 AU, respectively. If we use the distance from the simultaneous fit as our best estimate and assume that the independent fits give two measurements of the same quantity, then the standard deviation is $4.6 \mathrm{AU}$. The derived fluxes at the shock are 1.7 $\pm 0.3\left(\mathrm{~m}^{2} \mathrm{~s} \text { sr } \mathrm{MeV} / \text { nucleon }\right)^{-1}$ for $7.1-17.1 \mathrm{MeV} /$ nucleon 


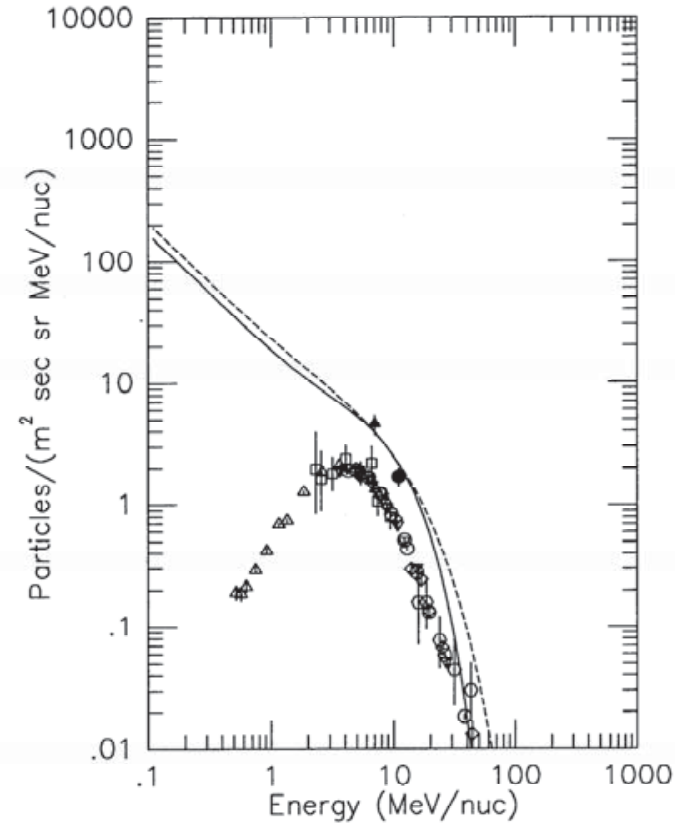

Figure 5a. Open symbols: "generic" ACR energy spectrum normalized to ACR O appropriate for the position of V2 during 1987 days 105-313 [from Cummings and Stone, 1990]. See Table 1 for the energy and flux scaling factors that were used to plot this composite spectrum. (Solid circle) Derived flux of ACR O at the shock. Solid triangle: Derived flux of ACR He at the shock, plotted to represent $\mathrm{O}$ using the scale factors from Table 1. (Solid curve) Calculated energy spectrum of ACR O at the shock using the model of Potgieter and Moraal [1988]. The shock distance was assumed to be 67 AU. The diffusion coefficient was of the form $\kappa \propto \beta$ below $400 \mathrm{MV}$ and given by $\kappa=1.48 \times 10^{17} \beta(\mathrm{R} / 1 \mathrm{MV})^{1.8} \mathrm{~cm}^{2} \mathrm{~s}^{-1}$ above 400 MV. (Dashed curve) Similar to solid curve except diffusion coefficient above $400 \mathrm{MV}$ was given by $\kappa=5.74 \times$ $10^{19} \beta(\mathrm{R} / 1 \mathrm{MV})^{1} \mathrm{~cm}^{2} \mathrm{~s}^{-1}$.

ACR oxygen and $4.3 \pm 0.8\left(\mathrm{~m}^{2} \mathrm{~s} \text { sr } \mathrm{MeV} / \text { nucleon }\right)^{-1}$ for 31-57 MeV/nucleon ACR helium.

\section{Discussion}

Cummings et al. [1984] have shown that the observed energy spectra of all the ACR elements have the same spectral shape when the intensity is plotted as a function of energy per nucleon. The energy spectrum of each individual element can be shifted by scale factors in energy/nucleon and intensity such that all spectra will overlay. This composite spectrum is referred to as the "generic" ACR energy spectrum and such a spectrum normalized to the ACR O energy spectrum at Voyager 2 for the period 1987 days 105 to 313 is shown in Figure 5a [Cummings and Stone, 1990]. The scale factors that produced this energy spectrum are shown in Table 1. Cummings et al. [1984] also showed that the energy scaling factor is a power law in mass, $A$, with the index determined by the rigidity dependence of the diffusion coefficient, where $\kappa \propto \beta R^{\gamma}$, with $\beta$ the particle velocity and $R$ the rigidity. The energy scaling factor of 6 between $\mathrm{He}$ and $\mathrm{O}$ in Table 1 corresponds to $\gamma=1.8$.

Also shown in Figure 5a are the derived fluxes of ACR O and ACR He at the shock. The ACR He shock flux has been scaled the same as the observed $\mathrm{He}$ in the generic spectrum, that is, the derived ACR He shock flux and energy have been divided by the scale factors of 0.945 and 6.01 , respectively, from Table 1 before plotting.

The two curves in Figure 5a represent two models for the ACR $\mathrm{O}$ spectra at the shock. Both were derived from a steady state spherically symmetric model with a strong shock (compression ratio of 4 ) developed by Potgieter and Moraal [1988] and exhibit the expected power law $\left(\propto E^{-1}\right)$ at low energies with an exponential roll off above a cutoff energy which depends on the radius of the shock and the diffusion coefficient. The solid curve in Figure $5 \mathrm{a}$ is the strong shock energy spectrum for $\kappa \propto \beta R^{1.8}$ and with the minimum value of the cutoff energy that is consistent with the derived shock fluxes and which gives positive modulation factors over the energy range of the observed ACR spectrum. A similar spectrum with a lower cutoff energy would have less flux at higher energies than observed. The dashed curve in Figure 5a, which is similar to the one for ACR O in Figure 2 of $I p$ [1990], represents the strong shock energy spectrum for $\kappa \propto \beta R$ and with the maximum value of the cutoff energy that is consistent with the derived shock fluxes and a modulation factor that generally decreases with increasing energy. A similar shock spectrum with a higher cutoff energy would require increasing modulation at higher energies to produce the observed spectrum. Comparison of the two spectra indicates little sensitivity to the assumed form of the diffusion coefficients for energies $\$ 20 \mathrm{MeV} /$ nucleon. The energy density corresponding to the dashed spectrum is $\sim 20 \%$ larger than that for the solid spectrum.

Although the spherically symmetric model does not include the effects of particle drifts inward along the heliospheric current sheet and therefore cannot account for the large latitudinal gradients observed in the outer heliosphere [Cummings et al., 1987, 1990b], it does yield generic shock spectra with the expected exponential roll off at high energies. As discussed below, the cutoff energies of the model spectra with $\kappa \propto \beta R^{1.8}$ scale with particle mass as required by the observations (Table 1 and Figure 5 b). As a result, we have used these model shock spectra to estimate the energy densities of the ACRs at the shock.

Christian et al. [1988] estimated that the peak intensity of ACR H observed at V2 during days $105-313$ of 1987 was 0.4 to 0.8 ( $^{2}$ s sr MeV) ${ }^{-1}$, subsequently refined to $0.67 \pm 0.18$ $\left(\mathrm{m}^{2} \mathrm{~s} \mathrm{sr} \mathrm{MeV}\right)^{-1}$ at $134 \mathrm{MeV}$ [Christian, 1989]. We can relate this value to the source flux of ACR $\mathrm{H}$ at the termination shock by using the same modulation factor as that deduced

Table 1. Energy and Flux Scaling Factors for Preparing the "Generic'" ACR O Energy Spectrum

\begin{tabular}{clcl}
\hline Element & $\begin{array}{c}\text { Symbol in } \\
\text { Figure 5a }\end{array}$ & $\begin{array}{c}\text { Energy } \\
\text { Factor }\end{array}$ & $\begin{array}{c}\text { Flux } \\
\text { Factor }\end{array}$ \\
\hline $\mathrm{He}$ & triangle & 6.01 & 0.945 \\
$\mathrm{C}$ & square & 1.71 & 0.0042 \\
$\mathrm{~N}$ & inverted triangle & 1.13 & 0.125 \\
$\mathrm{O}$ & circle & 1.00 & 1.00 \\
$\mathrm{Ne}$ & diamond & 0.85 & 0.08 \\
$\mathrm{Ar}$ & hexagon & 0.35 & 0.026 \\
\hline
\end{tabular}

The observed energies and fluxes are divided by the factors shown to yield the spectrum shown in Fig. 5a [see Cummings and Stone, 1990]. 


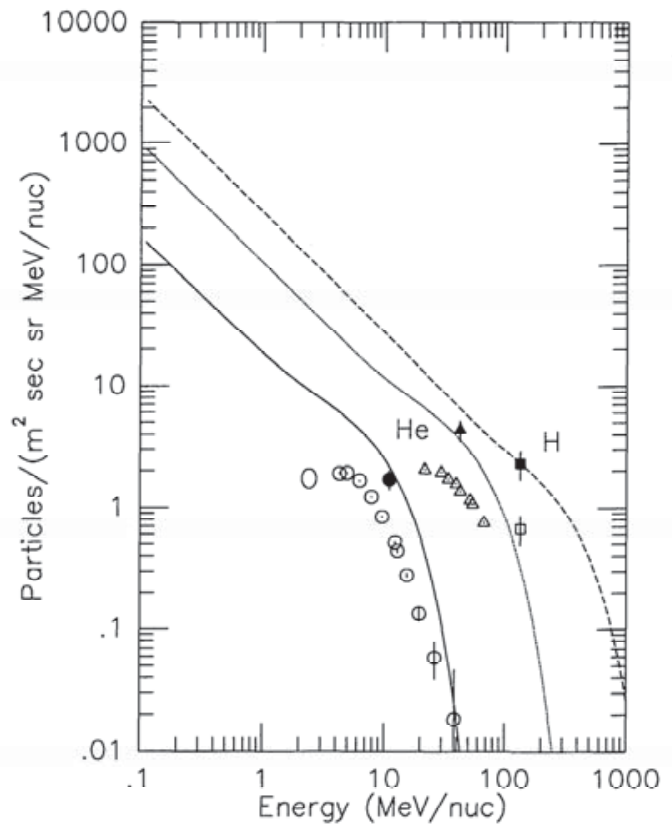

Figure 5b. Derived fluxes of ACR oxygen (solid circle), ACR helium (solid triangle), and ACR H (solid square) at the position of the termination shock (67 AU). The open points are the observed energy spectra of the ACRs at the position of V2 in 1987 days 105-313. The solid curve is the estimated energy spectrum of ACR O at the shock (identical to solid curve in Figure 5a). The dashed and dotted curves represent the expected energy spectra of ACR $\mathrm{H}$ and $\mathrm{He}$, respectively, at the shock and are calculated from the model of Potgieter and Moraal [1988] as described in the text.

for the generic ACR spectrum at the energy of its maximum intensity. From Figure 5a the maximum intensity of ACR O at V2 is 2.0 ( $\left.^{2} \mathrm{~s} \mathrm{sr} \mathrm{MeV/nucleon}\right)^{-1}$ at $3.6 \mathrm{MeV} /$ nucleon for the period 1987 days 105-313. From Figure 5a we estimate the flux of ACR O at the termination shock at $3.6 \mathrm{MeV} /$ nucleon (solid curve) to be $6.8\left(\mathrm{~m}^{2} \mathrm{~s} \text { sr MeV/nucleon) }\right)^{-1}$, yielding a modulation factor of 3.4. Applying this factor to the peak ACR $\mathrm{H}$ intensity observed at V2 yields an estimated flux of ACR $\mathrm{H}$ at the termination shock of $2.3 \pm 0.6$ $\left(\mathrm{m}^{2} \mathrm{~s} \mathrm{sr} \mathrm{MeV}\right)^{-1}$ at $134 \mathrm{MeV}$. In Figure 5b we show this flux as well as the spectra of ACR $\mathrm{H}$ and $\mathrm{He}$ at the termination shock calculated using the same parameters as that for the solid curve (ACR O) of Figure 5a. The calculated He curve has been normalized to give the same modulation factor at the peak energy of $21.7 \mathrm{MeV} /$ nucleon as that observed for ACR O at its peak energy of $3.6 \mathrm{MeV} /$ nucleon. The $\mathrm{H}$ curve has been normalized to pass through the deduced shock flux at $134 \mathrm{MeV}$. We also show in Figure 5b the energy spectrum of ACR O at the shock from Figure 5a (solid curve). The open data points represent the observed ACR fluxes of $\mathrm{O}$, $\mathrm{He}$, and $\mathrm{H}$ from V2 during the period 1987 days 105-313.

From these calculated strong shock energy spectra we estimate that the energy densities $\left(\mathrm{eV} / \mathrm{cm}^{3}\right)$ of the $\mathrm{H}^{+}, \mathrm{He}^{+}$, and $\mathrm{O}^{+} \mathrm{ACR}$ ions at the termination shock are $0.015 \pm$ $0.004,0.010 \pm 0.002$, and $0.003 \pm 0.001$, respectively. These three ions are expected to be the dominant contributors to the energy density of the ACRs at the termination shock. Thus the total energy density in anomalous cosmic rays at the termination shock is $0.028 \pm 0.005 \mathrm{eV} / \mathrm{cm}^{3}$ (or $0.013 \pm$ $0.002 \mathrm{eV} / \mathrm{cm}^{3}$ in the absence of ACR H). At $23 \mathrm{AU}$ the observed energy density of the solar wind at low heliographic latitudes in 1987 is $\sim 16 \mathrm{eV} / \mathrm{cm}^{3}$ (derived from Figure 1 of Grzedzielski and Lazarus [1993]), corresponding to $\sim 1.9$ $\mathrm{eV} / \mathrm{cm}^{3}$ at $67 \mathrm{AU}$. Thus for a strong shock energy spectrum, the energy density of the ACRs at the shock is $\sim 2 \%$ of the solar wind energy density (or $\sim 1 \%$ in the absence of ACR $\mathrm{H})$, indicating that the shock dissipation is not dominated by the acceleration of ACRs. The energy density associated with the dashed strong shock energy spectrum in Figure 5a would be only $\sim 20 \%$ larger.

P10 was between 39 and 44 AU during the 1986.7-1988.5 time period. Our result places the shock $\sim 23$ AU beyond P10 at the end of the period. It is expected that the shock location will vary in response to pressure variations in the solar wind. During most of the time period of this study these pressure variations are small [Grzedzielski and Lazarus, 1993] and hence large excursions of the shock are not expected. Recent work by Whang and Burlaga [1993] suggests that the radial position of the shock decreased by $\sim 10 \%$ during the time period of our study. The uncertainty on our estimate allows for a total range of variation of $\sim 15 \%$, within the range estimated by Whang and Burlaga [1933]. Our results suggest that during 1997 when both P10 and V1 will be beyond $67 \mathrm{AU}$, they may be in a position to observe the termination shock as it moves inward with the approaching solar minimum.

Acknowledgments. We are grateful to J. T. Hoeksema for providing the neutral sheet tilt data in machine readable form. This work was supported in part by NASA under contract NAS7-918 and grant NAGW-1919.

The Editor thanks L. A. Fisk and M. A. Forman for their assistance in evaluating this paper.

\section{References}

Adams, J. H., Jr., et al., The charge state of the anomalous component of cosmic rays, Astrophys. J. Lett., 375, L45, 1991.

Christian, E. R., Evidence for anomalous cosmic-ray hydrogen, Ph.D thesis, Calif. Inst. of Technol., Pasadena, 1989.

Christian, E. R., A. C. Cummings, and E. C. Stone, Evidence for anomalous cosmic-ray hydrogen, Astrophys. J. Lett., 334, L77, 1988.

Christian, E. R., A. C. Cummings, and E. C. Stone, Evidence for anomalous cosmic ray hydrogen, Proc. Int. Conf. Cosmic Ray 21st, 6, 186, 1990.

Cummings, A. C., and E. C. Stone, Elemental composition of the very local interstellar medium as deduced from observations of anomalous cosmic rays, Proc. Int. Conf. Cosmic Ray 21 st, 6, 202, 1990.

Cummings, A. C., E. C. Stone, and W. R. Webber, Evidence that the anomalous cosmic-ray component is singly ionized, Astrophys. J. Lett., 287, L99, 1984.

Cummings, A. C., E. C. Stone, and W. R. Webber, Latitudinal and radial gradients of anomalous and galactic cosmic rays in the outer heliosphere, Geophys. Res. Lett., 14, 174, 1987.

Cummings, A. C., R. A. Mewaldt, E. C. Stone, and W. R. Webber, Radial and latitudinal gradients of anomalous cosmic-ray oxygen and helium from 1 to $\sim 41 \mathrm{AU}$, Proc. Int. Conf. Cosmic Ray 21st, 6, 206, 1990a.

Cummings, A. C., E. C. Stone, and W. R. Webber, Time variation of radial and latitudinal gradients of anomalous cosmic ray oxygen in the outer heliosphere, Proc. Int. Conf. Cosmic Ray 21 st, 6, 190, 1990b.

Cummings, A. C., E. C. Stone, and W. R. Webber, Estimate of the distance to the solar wind termination shock from gradients of anomalous cosmic ray oxygen, J. Geophys. Res., 98, 15,165, 1993a.

Cummings, A. C., E. C. Stone, and W. R. Webber, The distance to 
the solar wind termination shock during 1986-1988, Proc. Int. Conf. Cosmic Ray 23rd, 3, 411, 1993b.

Fisk, L. A., B. Kozlovsky, and R. Ramaty, An interpretation of the observed oxygen and nitrogen enhancements in low-energy cosmic rays, Astrophys. J. Lett., 190, L35, 1974.

Geiss, J., G. Gloeckler, U. Mall, R. von Steiger, A. B. Galvin, and K. W. Olgivie, Interstellar oxygen, nitrogen, and neon in the heliosphere, Astron. Astrophys., in press, 1994.

Gloeckler, G., J. Geiss, H. Balsiger, L. A. Fisk, A. B. Galvin, F. M. Ipavich, K. W. Ogilvie, R. von Steiger, and B. Wilken, Detection of interstellar pick-up hydrogen in the solar system, Science, 261, 70, 1993.

Grzedzielski, S., and A. J. Lazarus, 2- to $30 \mathrm{kHz}$ continuum emissions as possible indications of global heliospheric "breathing," J. Geophys. Res., 98, 5551, 1993.

Hoeksema, J. T., Large-scale structure of the heliospheric magnetic field: 1976-1991, Proc. Solar Wind, 7, 191, 1992.

Ip, W.-H., On using the ACR to probe the LISM/heliosphere interface, in Physics of the Outer Heliosphere, Cospar Colloq. Ser., edited by S. Grzedielski and D. E. Page, p. 307, Pergamon, New York, 1990.

Jokipii, J. R., The anomalous component of cosmic rays, in Physics of the Outer Heliosphere, Cospar Colloq. Ser., edited by S. Grzedielski and D. E. Page, p. 169, Pergamon, New York, 1990. Lopate, C., and R. B. McKibben, The ${ }^{2} \mathrm{H} /{ }^{1} \mathrm{H}$ ratio as a test for the presence of anomalous protons at Pioneer 10 near solar minimum in 1987, Proc. Int. Conf. Cosmic Ray 22nd, 3, 390, 1991.

McDonald, F. B., N. Lal, J. H. Trainor, M. A. I. Van Hollebeke, and W. R. Webber, Observations of galactic cosmic-ray energy spectra between 1 and 9 AU, Astrophys. J., 216, 930, 1977.

Mewaldt, R. A., Temporal variations of anomalous cosmic rays and further evidence for anomalous cosmic ray hydrogen, Proc. Int. Conf. Cosmic Ray 21st, 6, 160, 1990.

Möbius, E., D. Hovestadt, B. Klecker, M. Scholer, G. Gloeckler, and F. M. Ipavich, Direct observation of $\mathrm{He}^{+}$pick-up ions of interstellar origin in the solar wind, Nature, 318, 426, 1985.

Pesses, M. E., J. R. Jokipii, and D. Eichler, Cosmic ray drift, shock wave acceleration, and the anomalous component of cosmic rays, Astrophys. J. Lett., 246, L85, 1981.

Potgieter, M. S., and H. Moraal, Acceleration of cosmic rays in the solar wind termination shock, I, A steady state technique in a spherically symmetric model, Astrophys. J., 330, 445, 1988.

Reinecke, J. P. L., and H. Moraal, On the form of the 1987 hydrogen spectrum in the outer heliosphere, Astrophys. J., 392, 272, 1992.

Seo, E. S., F. B. McDonald, N. Lal, and W. R. Webber, Measurement of the cosmic ray $\mathrm{H}$ and $\mathrm{He}$ isotopes at $23 \mathrm{AU}$, Proc. Int. Conf. Cosmic Ray 23rd, 1, 507, 1993.

Stone, E. C., R. E. Vogt, F. B. McDonald, B. J. Teegarden, J. H. Trainor, J. R. Jokipii, and W. R. Webber, Cosmic ray investigation for the Voyager missions: Energetic particle studies in the outer heliosphere-and beyond, Space Sci. Rev., 21, 355, 1977.

Whang, Y. C., and L. F. Burlaga, Termination shock: Solar cycle variations of location and speed, J. Geophys. Res., 98, 15221, 1993.

A. C. Cummings and E. C. Stone, California Institute of Technology, Pasadena,CA91125. (e-mail: Internet ace@citsrl.srl.caltech. edu; Internet ecs@citsrl.srl.caltech.edu)

W. R. Webber, New Mexico State University, Las Cruces, NM 88003. (e-mail: Internet bwebber@nmsu.edu)

(Received October 22, 1993; revised January 21, 1994; accepted February 21, 1994.) 